\title{
Optically induced electrostriction modes in a nanoparticle of a uniformly charged electret
}

\author{
S.I. Bastrukov1 1 , 2 I.V. Molodtsova ${ }^{2}$, Pik-Yin Lai $^{3}$ \\ 1 Department of Physics and Institute of Astronomy, \\ National Tsing Hua University, Hsinchu, 30013 Taiwan \\ 2 Laboratory of Informational Technologies, \\ Joint Institute for Nuclear Research, 141980 Dubna, Russia \\ ${ }^{3}$ Department of Physics and Center of Complex Systems, \\ National Central University, Chung-li, 320 Taiwan
}

\begin{abstract}
The electromagnetic response of a nanoparticle of an ion-doped polymeric elastic insulator, commonly called as an electret, is considered in the continuum model of a uniformly charged elastic sphere. The spectral formulae for the frequency of optically induced spheroidal and torsional shear oscillations driven by bulk force of elastic and dielectric stresses are obtained in analytic form. Particular attention is given to relaxation dielectric mode of the electrostriction response and its stability in the lowest quadrupole mode. The practical usefulness of ultrafine particles of electrets as a biolabels capable of accumulating likely-charged inclusions uniformly dispersed over the spherical volume of an elastic matrix is briefly discussed.
\end{abstract}

Key words: nanoparticle, ion-doped polymeric electret, biolabels

\section{Introduction}

The phenomenological continuum-mechanical models of vibrating liquid drop and solid globe provide proper account of gross features of resonance-like excitations in electromagnetic response of ultra fine particles. The spectra of these excitations are currently measured by methods of Raman scattering and inelastically scattered neutrons. The physical idea underlying these models is to identify the frequency of ac field incident on a nanoparticle with frequency of material oscillations in the particle regarded as a spherical piece of either liquid or solid continuous medium. A canonical example is fluid-mechanically computed Mie's spectral formula for the frequency of surface plasmons in nanoparticles of highly conducting

\footnotetext{
${ }^{1}$ Corresponding author, e-mail: bast@phys.nthu.edu.tw
} 
metals like silver and gold [1-5]. Another example is the solid-mechanically calculated frequency spectra of optically induced elastic oscillations, acoustic phonons, in a nanoparticle of a dielectric solid [6-8].

In this work we consider a model of resonant response of a nanoparticle of charged electret (insulating elastic matrix doped by likely-charged ions homogeneously distributed over spherical volume) responding to electromagnetic load by shear electro-mechanical fluctuations which are described in the approximation of a uniformly charged elastic continuum. To illuminate the difference between the electric reactive forces driven by optically-induced electro-mechanical vibrations in a nanoparticle of a uniformly charged electret (dielectric material) and plasma oscillations of free electrons against immobilized ions in a nanoparticle of noble metals (highly conducting materials), in Sec.2 a brief outline is given of classical electron theory of the surface plasmons and solid-mechanical theory of acoustic phonons. In Sec.3, the frequency spectra of electrostriction spheroidal and torsional vibrational modes in the ion-doped dielectric nanoparticle of electret are obtained followed by discussion of electro-elastic stability of such a nanoparticle to optically induced distortions, the issue of crucial importance to their use as biolabels. The results are briefly summarized in Sec.4.

\section{Resonance frequencies of surface plasmons and acous- tic phonons}

Within the framework of the classical electron theory of metals, the low-frequency electromagnetic response of metallic nanoparticle to long-wavelength ac field is modeled by oscillations of electron liquid against crystal lattice treated as a static uniformly charged ionic background and described by coupled equations of electron fluid-mechanics and ionic electrostatics

$$
\rho \frac{\partial \delta \mathbf{v}}{\partial t}=\rho_{e} \delta \mathbf{E}, \quad \rho=m_{e}^{*} n_{e}, \quad \rho_{e}=-e n_{e}
$$

The emergence of Coulomb restoring force is attributed to a small amplitude fluctuations of intrinsic electric field $\delta \mathbf{E}$ (between free electrons and ions) superimposed on electrostatic field of ionic jellium, $\mathbf{E}_{0}$ which is computed from the equation $\nabla \cdot \mathbf{E}_{0}=4 \pi \rho_{i}$, where $\rho_{i}=e n_{i}$; the particle number of electrons $n_{e}$ equals to that of ions $n_{i}$ due to electro-neutrality of metal nanoparticle. The effective mass of electron $m_{e}^{*}$ is unique to each specific metal. The effect of resonance photo-absorption emerges when the frequency of incident on particle ac field $\delta \mathbf{E}$ becomes equal to the frequency of oscillations of the velocity $\delta \mathbf{v}$ of electron flow obeying the vector Laplace equation:

$$
\nabla^{2} \delta \mathbf{E}=0, \quad \nabla^{2} \delta \mathbf{v}=0
$$


These equations can be thought of as long wavelength limit of the Helmholtz equation for standing waves and describing electromagnetic response by long wavelength vibrations of electric field and electron flow, Rayleigh regime. In this regime the frequency of oscillations of free electrons against ionic background can be computed by the energy method which is based on the equation of energy balance

$$
\frac{\partial}{\partial t} \int \frac{\rho \delta \mathbf{v}^{2}}{2} d \mathcal{V}=\int \rho_{e}(\delta \mathbf{v} \cdot \delta \mathbf{E}) d \mathcal{V} .
$$

The frequency of surface plasmons as a function of multipole degree $\ell$ is given by the wellknown Mie spectral formula [1-4]:

$$
\omega_{\mathrm{Mie}}^{2}(\ell)=\omega_{p}^{2} \frac{\ell}{2 \ell+1} \quad \omega_{p}^{2}=\frac{4 \pi n_{e} e^{2}}{m_{e}^{*}} \quad \ell \geq 1 .
$$

The dipole surface plasmon resonance whose energy is given by, $E_{\mathrm{Mie}}(\ell=1)=\hbar \omega_{p} / \sqrt{3}$, is well singled out in the cross sections of photo-absorption by silver and gold nanoparticles.

Also relevant to our further discussion is the continuum, solid-mechanical, description of acoustic phonons, quanta of low-frequency elastic oscillations of solid. The mathematical treatment of such vibrations rests on canonical equation of solid-mechanics for the field of material distortions $u_{i}(\mathbf{r}, t)$

$$
\rho \ddot{u}_{i}=\nabla_{k} \sigma_{i k}, \quad \sigma_{i k}=2 \mu u_{i k}, \quad u_{i k}=\frac{1}{2}\left[\nabla_{i} u_{k}+\nabla_{k} u_{i}\right], \quad u_{k k}=\nabla_{k} u_{k}=0 .
$$

where $\rho$ stands for the density the shear modulus $\mu$ relates applied shear stresses $\sigma_{i k}$ and resulting shear or strains $u_{i k}$ in accord with the law of Hooke. The conservation of energy is controlled by equation

$$
\frac{\partial}{\partial t} \int \frac{\rho \dot{u}^{2}}{2} d \mathcal{V}=-\int \sigma_{i k} \dot{u}_{i k} d \mathcal{V}, \quad \dot{u}_{i k}=\frac{1}{2}\left[\nabla_{i} \dot{u}_{k}+\nabla_{k} \dot{u}_{i}\right] .
$$

The fluctuating fields of material displacements $u_{i}(\mathbf{r}, t)$ and strains $u_{i k}(\mathbf{r}, t)$ can be conveniently represented in the following separable form

$$
\mathbf{u}(\mathbf{r}, t)=\mathbf{a}(\mathbf{r}) \alpha(t), \quad u_{i k}(\mathbf{r}, t)=a_{i k}(\mathbf{r}) \alpha(t), \quad a_{i k}=\frac{1}{2}\left[\nabla_{i} a_{k}+\nabla_{k} a_{i}\right]
$$

with help of which the equation of energy balance (6) is reduced to equation for $\alpha(t)$ having the form of standard equation of linear oscillations:

$$
M \ddot{\alpha}(t)+K \alpha(t)=0, \quad M=\int \rho a_{i}(\mathbf{r}) a_{i}(\mathbf{r}) d \mathcal{V}, \quad K=2 \int \mu a_{i k}(\mathbf{r}) a_{i k}(\mathbf{r}) d \mathcal{V} .
$$

In Rayleigh's regime, the vibrational response of an elastic sphere is characterized by two fundamental modes of shear elastic oscillations of the nodeless fields of material displacements which are specified by two fundamental solutions of the vector Laplace equation

$$
\nabla^{2} \mathbf{u}(\mathbf{r}, t)=0 \quad \rightarrow \quad \nabla^{2} \mathbf{a}(\mathbf{r})=0
$$


The instantaneous displacements in spheroidal mode of irrotational oscillations $-\mathbf{a}_{s}$ is given by poloidal field and in torsional mode of differentially rotational oscillations $-\mathbf{a}_{t}$ by the toroidal fields, respectively [7]:

$$
\mathbf{a}_{s}=\nabla \times \nabla \times(\mathbf{r} \chi), \quad \mathbf{a}_{t}=\nabla \times(\mathbf{r} \chi), \quad \chi(\mathbf{r})=\mathcal{N}_{\ell} r^{\ell} P_{\ell}(\cos \theta)
$$

Henceforth $P_{\ell}(\cos \theta)$ stands for the Legendre polynomial of multipole degree $\ell$ and $\mathcal{N}_{\ell}$ is the arbitrary constant. The spectral equations for the frequencies of the even parity spheroidal $\omega_{s}$ and the odd parity torsional $\omega_{t}$ modes of acoustic phonons are given by [ 6 , 7 ]:

$$
\begin{aligned}
& \omega_{s}(\ell)=\omega_{0}[2(2 \ell+1)(\ell-1)]^{1 / 2}, \quad \omega_{t}(\ell)=\omega_{0}[(2 \ell+3)(\ell-1)]^{1 / 2}, \quad \ell \geq 2, \\
& \omega_{0}=\frac{c_{t}}{R}, \quad c_{t}=\sqrt{\frac{\mu}{\rho}}
\end{aligned}
$$

where $c_{t}$ is the speed of elastic shear wave in the material bulk and $R$ stands for the radius of nanoparticle. These last spectral equations are central to spectroscopic analysis of optical modes excited by inelastically scattered x-rays and neutrons. The purpose of the above outlined models is to illuminate similarity and difference between spectral equations for the frequencies of surface plasmons, equation (5), and the frequency spectra of electrostriction modes in a nanoparticle of a uniformly charged electret which is the main subject of the next section.

\section{Electrostriction mode in optical response of nanopar- ticle of a uniformly charged electret}

The optically-induced, by ac electromagnetic field, electro-mechanical distortions in the volume of uniformly charged electret are characterized by the tensor of dielectric stresses (e.g., [9]):

$$
p_{i k}=\frac{1}{8 \pi}\left[E_{i} \delta D_{k}+E_{k} \delta D_{i}-\left(E_{j} \delta D_{j}\right) \delta_{i k}\right]
$$

where $E_{i}$ are components of electrostatic field produced in the particle volume by extraneous charge uniformly distributed with the charge density $\rho_{e}$ :

$$
\nabla \cdot \mathbf{E}=4 \pi \frac{\rho_{e}}{\epsilon}, \quad \nabla \times \mathbf{E}=0
$$

whose solution is well known:

$$
\mathbf{E}(\mathbf{r})=-\nabla \Phi(\mathbf{r}), \quad \Phi(r)=-\frac{2 \pi}{3 \epsilon} \rho_{e}\left(r^{2}-3 R^{2}\right), \quad\left[E_{r}=\frac{4 \pi}{3} \frac{\rho_{e}}{\epsilon} r, \quad E_{\theta}=0, \quad E_{\phi}=0\right] .
$$


The electro-mechanical effect is described by constituting equation 2

$$
\delta D_{i}=-4 \pi \rho_{e} u_{i}
$$

showing that optically induced fluctuations of dielectric induction $\delta D_{i}$ are linearly proportional to material displacements $u_{i}$ and inextricably related to the storage of extraneous charge uniformly dispersed with density $\rho_{e}$ over the sample volume; note, the dielectric materials can accommodate only extraneous charge.

\subsection{Dielectric relaxation mode}

We confine our analysis to the Rayleigh's regime of optical perturbation resulting in noncompressional fluctuations of the electret material (the charge density remains unchanged $\delta \rho_{e}=-\rho_{e} \nabla_{k} u_{k}=0$ ) which are described by nodeless field of material displacements $u_{i}$ obeying the vector Laplace equation, $\nabla^{2} \mathbf{u}=0$. The purpose of this subsection is to elucidate the difference between plasma oscillations of free electrons against ionic jellium in a metal nanoparticle which are restored by electrostatic force of the form $f_{i}=\rho_{e} E_{i}$, Eq.(11), and the shear oscillations in a nanoparticle of uniformly charged electret which are restored by reactive force of dielectric stresses $f_{i}^{\prime}=\nabla_{k} p_{i k}$ which are governed by equations

$$
\rho \ddot{u}_{i}=\nabla_{k} p_{i k}, \quad \frac{\partial}{\partial t} \int \frac{\rho \dot{u}^{2}}{2} d \mathcal{V}=-\int p_{i j} \dot{u}_{i j} d \mathcal{V}, \dot{u}_{i k}=\frac{1}{2}\left[\nabla_{i} \dot{u}_{k}+\nabla_{k} \dot{u}_{i}\right], u_{k k}=0 .
$$

The rightmost equation shows that optically induced dielectric stresses $p_{i k}$ are accompanied by shear deformations $u_{i k}$. The eigenfrequency can be computed with help of the above expounded Rayleigh's energy method whose key point is the separable representation of fluctuating material displacements and strains in the form given by equations (7). Then, for the perturbation-induced dielectric induction one has

$$
\delta D_{i}(\mathbf{r}, t)=-4 \pi \rho_{e} a_{i}(\mathbf{r}) \alpha(t)
$$

and the tensor of dielectric stress is given by

$$
p_{i k}(\mathbf{r}, t)=\left[\tau_{i k}(\mathbf{r})-\frac{1}{2} \tau_{j j} \delta_{i k}\right] \alpha(t), \quad \tau_{i k}(\mathbf{r})=-\rho_{e}\left[E_{i}(\mathbf{r}) a_{k}(\mathbf{r})+E_{k}(\mathbf{r}) a_{i}(\mathbf{r})\right] .
$$

${ }^{2}$ The constitutive equation for $\delta D_{i}$ is compatible with the Maxwell equation $\nabla \times \delta \mathbf{H}=(4 \pi / c) \delta \mathbf{j}+(1 / c) \delta \dot{\mathbf{D}}$. Applying to this latter equation operator of divergence and taking into account that $\delta \mathbf{j}=\rho_{e} \delta \mathbf{v}$ and the continuity equation of the charge conservation $\delta \dot{\rho}_{e}=-\nabla \delta \mathbf{j}$, we obtain $\delta \dot{\mathbf{D}}=-4 \pi \rho_{e} \delta \mathbf{v}$. Bearing in mind that $\delta \mathbf{v}=\dot{\mathbf{u}}$ and eliminating in the last equation the time derivative one arrives at (16). 
Substitution (7), (18) and (19) into equation of energy balance in (17) leads to

$$
\begin{aligned}
& \frac{d H}{d t}=0 \quad H=\frac{M \dot{\alpha}^{2}}{2}+\frac{K_{d} \alpha^{2}}{2} \rightarrow \ddot{\alpha}(t)+\omega^{2} \alpha(t)=0, \quad \omega^{2}=\frac{K_{d}}{M} \\
& M=\int \rho a_{i} a_{i} d \mathcal{V}, \quad K_{d}=\int \tau_{i k}(\mathbf{r}) a_{i k}(\mathbf{r}) d \mathcal{V} \\
& \tau_{i k}(\mathbf{r})=-\rho_{e}\left[E_{i}(\mathbf{r}) a_{k}(\mathbf{r})+E_{k}(\mathbf{r}) a_{i}(\mathbf{r})\right], \quad a_{i k}=\frac{1}{2}\left[\nabla_{i} a_{k}+\nabla_{k} a_{i}\right] .
\end{aligned}
$$

The fact that such response is accompanied by internal shear deformations suggests that dielectric modes in question can be specified in a manner of vibrational modes in an elastic sphere, that is, as spheroidal and torsional ones.

Dielectric spheroidal mode. In this positive parity mode the displacements are described by the poloidal (polar) vector field

$$
\mathbf{a}_{s}=\mathcal{N}_{\ell} \nabla \times \nabla \times \mathbf{r} r^{\ell} P_{\ell}(\cos \theta)
$$

For the inertia, stiffness and frequency $\omega_{d s}$ of spheroidal dielectric mode we obtain

$$
\begin{aligned}
& M^{s}=4 \pi \rho \mathcal{N}_{\ell}^{2} R^{2 \ell+1} \frac{\ell(\ell+1)^{2}}{(2 \ell+1)}, \quad K_{d}^{s}=-\frac{32 \pi^{2}}{3} \frac{\rho_{e}^{2}}{\epsilon} \mathcal{N}_{\ell}^{2} R^{2 \ell+1} \frac{\ell(\ell+1)\left(\ell^{2}-1\right)}{(2 \ell+1)}, \\
& \omega_{d s}^{2}(\ell)=-\frac{2}{3} \omega_{d}^{2}(\ell-1), \quad \omega_{d}^{2}=\frac{4 \pi}{\epsilon} \frac{\rho_{e}^{2}}{\rho}
\end{aligned}
$$

where $\omega_{d}$ is the natural unit of dielectric fluctuations.

Dielectric toroidal mode. The material displacements in negative parity torsional mode are described by the axial toroidal (axial) vector field

$$
\mathbf{a}_{t}=\mathcal{N}_{\ell} \nabla \times \mathbf{r} r^{\ell} P_{\ell}(\cos \theta)
$$

Computation of the the inertia, stiffness and frequency $\omega_{d t}$ of torsional dielectric mode yields

$$
\begin{aligned}
& M^{t}=4 \pi \rho \mathcal{N}_{\ell}^{2} R^{2 \ell+3} \frac{\ell(\ell+1)}{(2 \ell+1)(2 \ell+3)}, \quad K_{d}^{t}=-\frac{16 \pi^{2}}{3} \frac{\rho_{e}^{2}}{\epsilon} \mathcal{N}_{\ell}^{2} R^{2 \ell+3} \frac{\ell\left(\ell^{2}-1\right)}{(2 \ell+1)(2 \ell+3)}, \\
& \omega_{d t}^{2}(\ell)=\frac{K_{t}}{M_{t}}=-\frac{1}{3} \omega_{d}^{2}(\ell-1), \quad \omega_{d}^{2}=\frac{4 \pi}{\epsilon} \frac{\rho_{e}^{2}}{\rho} .
\end{aligned}
$$

The obtained spectral equations represent one of the main newly obtained result of the presented theory showing that optical response of nanoparticle of electret are characterized by two different in parity modes, even-parity spheroidal dielectric mode and odd-parity torsion one. The basic dielectric frequency $\omega_{d}$ depends upon the dielectric constant $\epsilon$ in such a way that in the limit $\epsilon \rightarrow \infty$, as is the case of conductors, $\omega_{d} \rightarrow 0$. This means that, 
contrary to the surface plasmons in a nanoparticle of a highly conducting noble metals, the electrostatic fluctuations in a nanoparticle of a uniformly charged electret are manifested as relaxation modes, not oscillatory. It is relevant to note that the somewhat different method of computing the frequency of nodeless electro-elastic vibrations of a uniformly charged dielectric globe that has been developed in [10, 11] leads to identical analytic estimates for the frequencies of considered dielectric modes. The negative sign of squared frequencies, highlighting relaxation nature of dielectric mode, indicates that nanoparticle is unstable to optically induced electro-mechanical distortions 3 . In the next section we consider oscillatory response of such particle which is governed by superposition of two restoring forces, namely, the constructive elastic restoring force whose strength is dominated by gradient in shear modulus

$$
f_{i}^{\prime \prime}=\nabla_{k} \sigma_{i k}=2 u_{i k} \nabla_{k} \mu(r)+2 \mu \nabla_{k} u_{i k} \approx 2 u_{i k} \nabla_{k} \mu, \quad \nabla_{k} u_{i k}=\nabla^{2} u_{i}=0 .
$$

and the above destructive force of dielectric stresses, $f_{i}^{\prime}=\nabla_{k} p_{i k}$ and and derive condition of stability which imposes severe constrain on the total charge and radius of the uniformly charged nanoparticle of electret which is of crucial importance to their practically useful applications.

\subsection{Frequencies of electrostriction modes}

The optically induced vibrations driven by combined the above forces of gradient-shear elastic stress $f^{\prime \prime}$ and dielectric stresses $f^{\prime}$ are governed the following equations of electro-

\footnotetext{
${ }^{3}$ The origin of this instability can be traced from equations of electro-elastic solid mechanics describing propagation of perturbations in bulk of uniformly charged electret with constant shear modulus $\mu$ which is given by

$$
\rho \ddot{u}_{i}=\nabla_{k} \sigma_{i k}+\nabla_{i} p_{i k}=\mu \nabla^{2} \mathbf{u}+(4 \pi / \epsilon) \rho_{e}^{2} \mathbf{u} .
$$

For the plane-wave form of externally induced displacements, $\mathbf{u}=\mathbf{u}_{t}^{0} \exp i(\mathbf{k r}-\omega t)$, the last equation leads to dispersion equation

$$
\omega^{2}=c_{t}^{2} k^{2}-\omega_{d}^{2}, \quad c_{t}^{2}=\frac{\mu}{\rho}, \quad \omega_{d}^{2}=\frac{4 \pi}{\epsilon} \frac{\rho_{e}^{2}}{\rho}
$$

where $c_{t}$ is the speed of transverse shear wave in the bulk electret and $\omega_{d}$ stands for the natural unit of frequency of dielectric oscillations. It follows that there is critical wave number $k_{c}$ (and corresponding wavelength $\lambda_{c}=2 \pi / k_{c}$ such that the electret material becomes electro-mechanically unstable when

$$
k<k_{c}=\sqrt{\frac{4 \pi \rho_{e}^{2}}{\epsilon \mu}}, \quad \lambda>\lambda_{c}=\sqrt{\frac{\pi \epsilon \mu}{\rho_{e}^{2}}} .
$$

Understandably that this instability owe its origin to the presence of extraneous charges.
} 
elastic dynamics and energy balance

$$
\begin{aligned}
& \rho \ddot{u}_{i}=\nabla_{k} \sigma_{i k}+\nabla_{i} p_{i k}=2 u_{i k} \nabla_{k} \mu+\frac{1}{8 \pi} \nabla_{k}\left[E_{i} \delta D_{k}+E_{k} \delta D_{i}\right], \quad \delta D_{i}=-4 \pi \rho_{e} u_{i}, \\
& \frac{\partial}{\partial t} \int \frac{\rho \dot{u}^{2}}{2} d \mathcal{V}=-2 \int\left(\nabla_{k} \mu\right) u_{i k} \dot{u}_{i} d \mathcal{V}-\int \rho_{e}\left[E_{i} u_{k}+E_{k} u_{i}\right] \dot{u}_{i k} d \mathcal{V}
\end{aligned}
$$

and making use of substitution $u_{i}(\mathbf{r}, t)=a_{i}(\mathbf{r}) \alpha(t)$ we obtain

$$
\begin{aligned}
& M \ddot{\alpha}+K \alpha=0, \quad M=\int \rho a_{i} a_{i} d \mathcal{V}, \quad K=K_{\mu}+K_{d}, \\
& K_{\mu}=2 \int\left(\nabla_{k} \mu\right) a_{i} a_{i k} d \mathcal{V}, \quad K_{d}=-\frac{1}{2} \int \rho_{e}\left[E_{i} a_{k}+E_{k} a_{i}\right]\left[\nabla_{i} a_{k}+\nabla_{k} a_{i}\right] d \mathcal{V} .
\end{aligned}
$$

The mass parameters in spheroidal and torsional modes are given by

$$
M^{s}=4 \pi \rho \mathcal{N}_{s}^{2} R^{2 \ell+1} \frac{\ell(\ell+1)^{2}}{(2 \ell+1)}, \quad M^{t}=4 \pi \rho \mathcal{N}_{t}^{2} R^{2 \ell+3} \frac{\ell(\ell+1)}{(2 \ell+1)(2 \ell+3)}
$$

and for the stiffness one has

$$
\begin{gathered}
K_{\mu}^{s}=8 \pi \mathcal{N}_{s}^{2} \ell(\ell-1) \int_{0}^{R}\left(\nabla_{r} \mu\right) r^{2 \ell-1} d r, \quad K_{d}^{s}=-\frac{32 \pi^{2}}{3} \frac{\rho_{e}^{2}}{\epsilon} \mathcal{N}_{s}^{2} R^{2 \ell+1} \frac{\ell(\ell+1)\left(\ell^{2}-1\right)}{(2 \ell+1)}, \\
K_{\mu}^{t}=4 \pi \mathcal{N}_{t}^{2} \frac{\ell\left(\ell^{2}-1\right)}{(2 \ell+1)} \int_{0}^{R}\left(\nabla_{r} \mu\right) r^{2 \ell+1} d r, \quad K_{d}^{t}=-\frac{16 \pi^{2}}{3} \frac{\rho_{e}^{2}}{\epsilon} \mathcal{N}_{t}^{2} R^{2 \ell+3} \frac{\ell\left(\ell^{2}-1\right)}{(2 \ell+1)(2 \ell+3)} .
\end{gathered}
$$

These equations provide a basis for computing frequency

$$
\omega^{2}=\frac{K_{\mu}+K_{d}}{M}
$$

of electrostriction vibrational response of a nanoparticles of uniformly charged electret with the non-homogeneous shear modulus profile.

As a representative example let us consider a case of nanoparticle with shear modulus profile given by

$$
\mu(r)=\mu\left[1-\left(\frac{r}{R}\right)\right]
$$

In this case the frequency of spheroidal electrostriction mode can be conveniently represented in the following form

$$
\begin{aligned}
\omega_{s}^{2} & =\omega_{e}^{2} 2(2 \ell+1)(\ell-1) \ell^{-1}-\frac{2}{3} \omega_{d}^{2}(\ell-1) \\
& =\omega_{e}^{2} 2(2 \ell+1)(\ell-1) \ell^{-1}\left[1-\frac{\ell}{3(2 \ell+1)} \beta\right], \quad \beta=\frac{\omega_{d}^{2}}{\omega_{e}^{2}}
\end{aligned}
$$


and for the torsional electrostriction mode as follows

$$
\begin{aligned}
\omega_{t}^{2} & =\omega_{e}^{2} \frac{(2 \ell+3)(\ell-1)}{2(\ell+1)}-\frac{1}{3} \omega_{d}^{2}(\ell-1) \\
& =\omega_{e}^{2} \frac{(2 \ell+3)(\ell-1)}{2(\ell+1)}\left[1-\frac{2(\ell+1)}{3(2 \ell+3)} \beta\right], \quad \beta=\frac{\omega_{d}^{2}}{\omega_{e}^{2}}
\end{aligned}
$$

One sees that the lowest overtone of both spheroidal and torsional modes is of quadrupole degree $\ell=2$. The absence of monopole $\ell=0$, breathing overtone, is the consequence of adapted approximation of incompressible matter. The dipole fields of both poloidal and toroidal displacements describe center-of-mass translation and rigid-body rotation, respectively, that is, the non-vibrational reaction of nanoparticle.

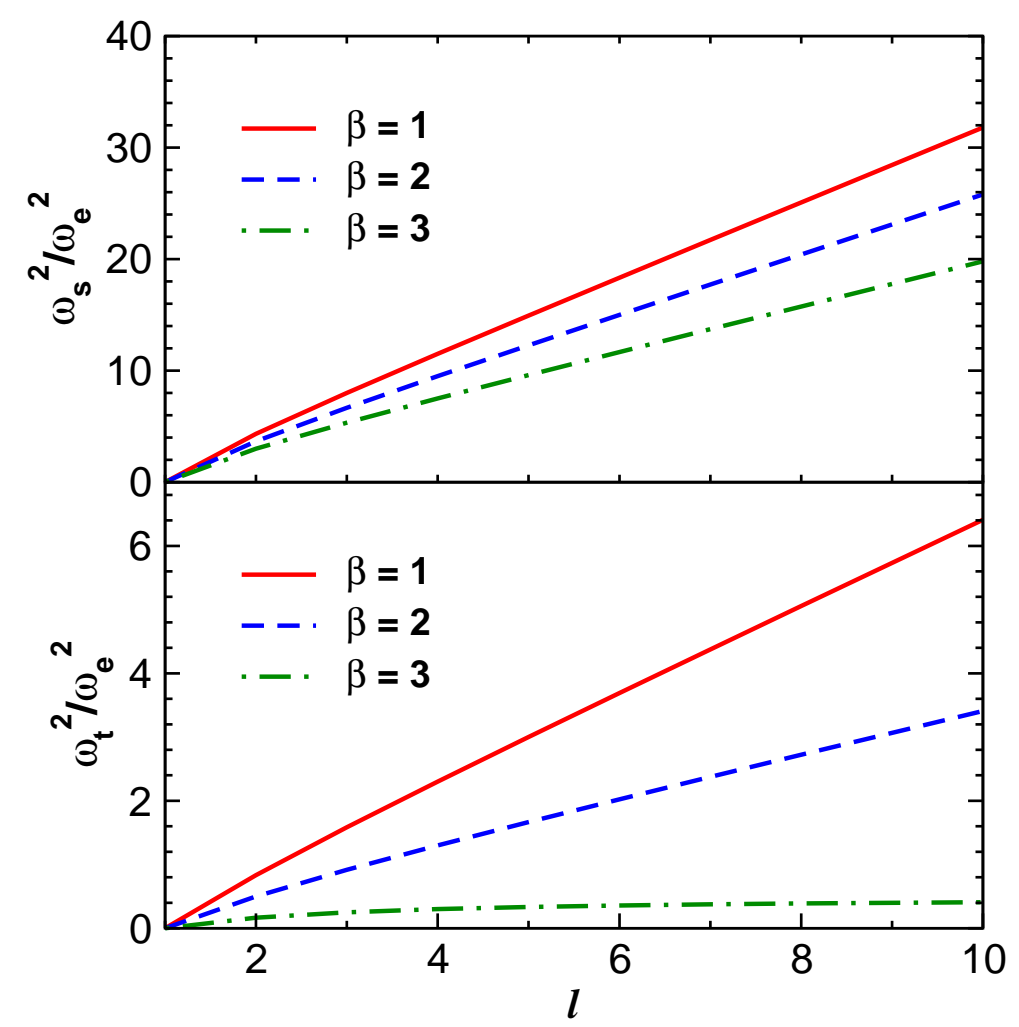

Figure 1: Ratio of the squared frequency of spheroidal (upper panel) and torsional (down panel) electrostriction modes to the squared frequency of elastic shear oscillations as a function of multipole degree at indicated values of parameter of stability $\beta$.

In Fig. 1 we plot the ratio $\omega_{s}^{2} / \omega_{e}^{2}$ and $\omega_{t}^{2} / \omega_{e}^{2}$ as functions of the multipole degree of spheroidal and torsional vibrations $\ell$, respectively, showing that the larger the $\ell$ the higher is the frequency. Also, this figure exhibits strong dependence of frequencies upon the parameter

$$
\beta=\frac{\omega_{d}^{2}}{\omega_{e}^{2}}=\frac{3}{4 \pi}(\epsilon \mu)^{-1} \frac{Q^{2}}{R^{4}}, \quad Q=\rho_{e} \mathcal{V}, \quad \mathcal{V}=(4 \pi / 3) R^{3}
$$


carrying information about total charge $Q$ accumulated by particle of radius $R$ and shows that the larger $\beta$ (the large the ratio $Q / R^{2}$ ) the lower is the frequency. The most conspicuous feature of electrostriction response in question is that the lowest, quadrupole, overtones become unstable when the parameter $\beta$ attains critical value $\beta=\beta_{c}$. Specifically, the spheroidal electrostriction vibrational mode becomes unstable, meaning $\omega_{s}(\ell=2)=0$, when

$$
\left[1-\frac{\ell}{3(2 \ell+1)} \beta\right]_{\ell=2}=0 \rightarrow \beta_{c}^{s}=\frac{15}{2} .
$$

The lowest quadrupole torsional electrostriction vibrational mode unstable, $\omega_{s}(\ell=2)=0$, when

$$
\left[1-\frac{2(\ell+1)}{3(2 \ell+3)} \beta\right]_{\ell=2}=0 \rightarrow \beta_{c}^{t}=\frac{7}{2}
$$

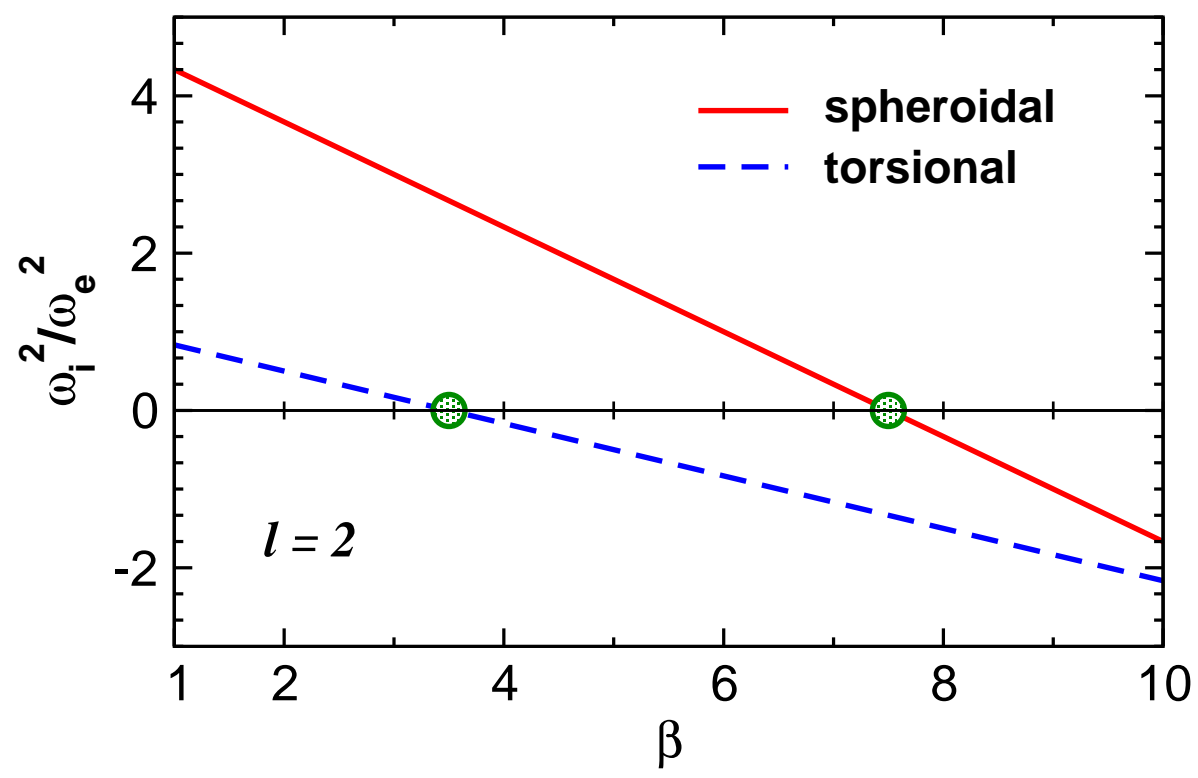

Figure 2: The frequency of quadrupole spheroidal and torsional electrostriction modes as a function of the stability parameter $\beta$. The circles on $0 \mathrm{X}$-axis mark values of $\beta$ at which the electrostriction mode becomes unstable undergoing transition from regime of oscillations to the relaxation regime.

In Fig. 2 we plot the ratio of squared frequencies of quadrupole, $\ell=2$, overtones of both spheroidal and torsion electrostriction modes as functions of the stability parameter $\beta$ by highlighting the above critical values of stability parameter $\beta_{c}$ by circles on the $0 \mathrm{X}$-axis. In this points the electrostriction modes undergo transition from the oscillatory regime to the relaxation regime. This leads us to conclude that nanoparticle of uniformly charged electret 
is stable to optically induced deformation oscillations when

$$
\frac{Q}{R^{2}}<C(\sqrt{\epsilon \mu})
$$

where constant $C$ falls in the range $5<C<10$; understandably that the lowest $\beta_{c}$ should be regarded as genuine critical value of this parameter. The practical usefulness of the above established conditions of instability is that it imposes severe constrain on the size and total charge accommodated by the electret nanoparticles and this must be taken into account in the process for technological fabrication. As a representative example, for the nano and micro dimensions $10^{-8}<R<10^{-6}$ m whose total charge is of the order of $Q \approx 10^{2} e \approx 10^{-16}$ (in SI units) from polymers with dielectric constant $1<\epsilon<15$, and shear modulus $10^{6}<\mu<10^{9}$ (in SI units), from above obtaned condition it follows that $10^{-4}<Q / R^{2}<1$. So that for ultra fine particles of nano sizes this condition is always fulfilled.

\section{Summary}

In this, somewhat technical in mathematical details, paper the theory of electrostriction response of nanoparticle of non-conducting elastic polymers capable of accumulating an extraneous charge to ac electromagnetic load has been developed. The prime purpose was to elucidate stability of such nanoparticles to optically induced distortions. This issue is of crucial importance to utilization of uniformly charged polymeric ultra fine particles (which are currently produced by jammed technique) as biolabels as well as for other hi-tech application. Based on general equations of electro-solid-mechanics appropriate for an isotropic electrets capable of accumulating likely-charged inclusions uniformly dispersed over the volume, the model of spherical particle of such a material undergoing optically induced spheroidal and torsional oscillations restored by superposition of two forces has been considered, namely, the constructive elastic force dominated by gradient of shear modulus and destructive force of dielectric stresses has been considered. It is that, contrary to nanoparticle to noble metals (responding to ac field by surface plasmon resonances which are independent upon particle size), the optical response of nanoparticles of uniformly charged electrets strongly depends upon their size and total accumulated charge. The conditions of electro-elastic stability of optically induced electrostriction spheroidal and torsional vibrational modes has been obtained showing that both modes of such response undergo transition from regime of oscillations to the relaxation regime. This condition highlights the most important difference between optically induced electrostatic fluctuations in the dielectric nanoparticle of electret which are of volume character and electrostatic, plasma, fluctuations in the metal nanoparticle which are of surface character: in this latter case these oscillations are stable whereas in the former 
case the electrostatic fluctuation can lead to disintegration of the electret nanoparticle by means of electric discharge. All these suggest if the condition of electro-elastic stability is fulfilled the optically induced electrostriction modes in question can be detected as resonant modes of photo absorbtion and the lowest overtones of these modes are of quadrupole degree.

\section{Acknowledgements}

This work is partly supported by NSC of Taiwan, under grants NSC- 97-2811-M-007-003.

\section{References}

[1] Ashley J C, Ferrell T L and Ritchie R H 1974 Phys. Rev. B 10554

[2] Bertsch G F and Broglia R A 1994 Oscillations in Finite Quantum Systems (Cambridge: Cambridge University Press)

[3] Kreibig U and Vollmer M 1994 Optical Properties of Metal Clusters (Berlin: Springer)

[4] Bastrukov S Lai P Y 2005 Phys. Lett. A 341207

[5] Bastrukov S Lai P Y 2006 Surf. Rev. Lett. 1381

[6] Bastrukov S 1994 Phys. Rev. E 493166

[7] Bastrukov S I, Cheng H K, Mişicu Ş, Molodtsova I V and Podgainy D V 2007 Int. J. Mod. Phys. A 223261

[8] Hernández-Rosas J, Picquart M, Haro-Poniatowski E, Jouanne M, Morhange J F, Kanehisa M, 2003 J. Phys.: Cond. Mat. 157481

[9] Lee H Y, Peng Y, Shkel Yu M, 2005 J. Appl. Phys. 98074104

[10] Bastrukov S and Podgainy D 1998 Physica A 250435

[11] Bastrukov S, Chervyakov A, Puzynin I and Podgainy D 1999 Progress in the Physics of Clusters eds. Chuev G, Lakhno V and Nefedov A (Singapore: World Scientific) p. 327 